\title{
Placenta related pathogenic factors for preeclampsia
}

\author{
Yuxuan Chen, Zhenyu Zhang* \\ Department of Obstetrics \& Gynecology, Beijing Chaoyang Hospital, Affiliated Hospital of Capital Medical University, Beijing, China \\ Email: *zhenyu2000@yahoo.com
}

Received 31 July 2012; revised 30 August 2012; accepted 10 September 2012

\begin{abstract}
Preeclampsia (PE) is a life-threatening complication of pregnancy. The precise origin of $P E$ remains obscure. Placenta has been considered to play a central role in its pathology. Here we present a brief overlook of placenta related pathogenic factors that might be involved in the pathology changes of PE. A series of factors that correlated with placenta pathology have been regarded attributed to the mechanism of the disease. Some of the factors may be confirmed to be useful biomarkers in the early prediction and monitoring of the disease in a future.
\end{abstract}

Keywords: Preeclampsia; Biomarker; Angiogenic Factors; Transthyretin

\section{INTRODUCTION}

$\mathrm{PE}$ is a major contributor to maternal and fetal/neonatal mortality and morbidity, affecting about 5\% - 8\% of pregnancy, but occurring up to $10 \%$ in China. In the mother, it can cause multi-system dysfunction including renal failure, hepatic failure, coagulopathy and central nervous system disorders. To the fetus, it may lead to fetal growth restriction, prematurity and perinatal death. However, an increasing number of women are involved in this severe complication. Currently, inadequate antenatal care has given to the pregnant women. There is a need for a widely applicable screening test that could permit early prediction and diagnosis of PE. Attention has turned towards identifying non-invasive, blood-borne or urinary maternal biomarkers that could predict the development and help to the monitoring of this severe complication of pregnancy. Understanding of the pathology mechanism for PE would enable a better conduction for this task [1-3].

Although the precise origin of PE remains unclear, the changes of placenta have been confirmed to play a middle role in pathology alternations of PE. Inadequate invasion of placental trophblast could be responsible for the origin of the disease. In normal conditions, the inva-

*Corresponding author. sion of trophblast causes the replacement of the muscular and elastic layers of the spiral arteries by fibrous tissue and then establishes a low-resistance fetoplacental blood supply. In PE, there could be an impaired tissue and arterial invasion by trophoblast cells, which may result in an unsuccessful transformation of spiral arteries and increased placental ischemia and necrosis. It has been reported that placenta ischemia may lead to an extra release of circulation toxin that might cause the pathophysiology alternations in PE [1,3-5].

To date, some factors have been reported to be related to the pathology changes of the disease, some of which have been regarded to be candidate markers of PE. We here present an overlook of some pathogenic factors correlated with placenta pathology changes in PE, which we hope could do a little conduction to the later researches in the future.

\section{PLACENTAL FACTORS}

\subsection{Transforming Growth Factor Beta Family}

Recently, transforming growth factor beta (TGF- $\beta$ ) has been confirmed to play a crucial role in placental development. The alternations of TGF- $\beta$ have been regarded as potential contributors to pregnancy induced hypertension. Attention has been turned to the exploration of contributions of TGF- $\beta$ and its regulators in PE [6,7].

\subsection{Soluble Endoglin}

Endoglin(Eng) is a co-receptor for transforming growth factor (TGF)- $\beta 1$ and TGF- $\beta 3$, which is highly expressed at endothelial cell membranes and syncytiotrophoblasts. Its main function is to modulate the TGF- $\beta$ signaling and involved in angiogenesis. Soluble Eng (sEng) is the extra part Eng molecule, acting as a circulating form of Eng, has been identified in normal pregnancy and PE condition. sEng has been confirmed to be a competitive inhibitor of TGF- $\beta$, thereby giving negative impact on angiogenesis [8]. A previous study has confirmed that high arterial pressure and vascular permeability could be induced by over-expressed sEng in pregnant rats [9]. In normal pregnancy, concentrations of sEng decreases 
between the first and second trimester, then raise during the last 2 month of normal gestation. In pregnancy ending with $\mathrm{PE}$, the levels of sEng goes down blunted between the second and third trimester, then increases earlier and sharper in the later part of pregnancy $[10,11]$. Therefore, it has been demonstrated that sEng levels are elevated in the sera of women superimposed with PE and appears to increase with the severity of the complication and gets to the highest in PE complicated with HELLP syndrome [12]. According to the previous study, levels of sEng are elevated $9-11$ weeks before the clinical symptoms present in women who developed PE. However, the changes of sEng levels may not specific for PE [11]. Pregnancy with in utero growth restriction (IUGR) without maternal symptoms may also characterized with elevated sEng levels, and it is more sensitive to $\mathrm{PE}$ women complicated with smaller gestation age (SGA) [11-13]. As above, It indicated that sEng may be associated with placental pathology, but alone might not serve as a marker for PE for a specific prediction can not be achieved. Further study with larger population is required to assess its role in pathology changes and clinical value.

\subsection{Inhibin $A$ and Activin $A$}

Inhibin A and activin A, are both glycoprotein mainly produced by the syncytiotrophoblast of the human placenta, belong to the TGF- $\beta$ family [14]. The second trimester levels of both Inhibin A and activin A have been shown elevated in women who subsequently develop PE, as early as 10 - 15 weeks of pregnancy prior to onset of the disease, and rapidly declined after $24 \mathrm{~h}$ following delivery $[15,16]$. Some researchers consider that changes of maternal serum levels of inhibin A and activin A may be correlated with placental pathology changes. It has been recently shown that oxidative stress stimulates activin A production and secretion from placenta and endothelial cells, in women with PE levels of activin A is significantly associated with markers for excessive oxidative stress $[16,17]$. That may provide an explanation for the higher levels of activin A in PE. As to inhibin $\mathrm{A}$, it has been reported inhibin $\mathrm{A}$ is higher in amniotic fluid, which has been considered correlated with the higher maternal serum inhibin A levels in women with PE. During PE, placenta may produces larger amount of inhibin A that enters maternal and the fetal circulation may cause an elevation in maternal serum and amniotic fluid inhibin A levels. In ShinYoung Kim's study, they consider that increased inhibin A levels in maternal circulation may be attributed to amniotic fluid inhibin A entering maternal circulation [17]. However, the mechanism of increased Inhibin A and activin A in PE is not yet fully understood, whether they have a role in the pathology of PE is still worth examing.

\section{TROPHOBLAST INVASION RELATED FACTORS}

Inadequate placental trophblast invasion has been confirmed to be key point in the pathology of PE, the mechanism of which has not been declared. Some circulating factors has already regarded correlated with the placental changes in PE. Here we give a introduction of some pathogenic factors that contribute to the inadequate placenta invasion in $\mathrm{PE}$, some of which could be candidate biomarkers of the disease [1-3].

\subsection{Angiogenic Factors}

Angiogenic factors have recently been regarded as a contributor to the pathogenesis of PE. Placenta is a rich source of these factors. Angiogenesis requires the interplay of angiogenic factors, include vascular endothelial growth factor (VEGF) and placental growth factor (PIGF) with their cognate receptors VEGF receptor-1 (VEGFR1 , which is alternatively called fms-like tyrosine kinase (flt)-1) and VEGFR-2 $[18,19]$. The imbalance of angiogenic factors have been confirmed to be attributed to inadequate trophoblast invasion and spiral artery remodeling, which can lead to hypoxia of placenta. It has also been proposed that maternal endothelial dysfunction in PE may be caused by the imbalance of levels of angiogenic factors $[3,20]$. Soluble flt-1(sflt-1) is a secreted splice variant of flt-1. Excessive sflt-1 induced hypertension, proteinuria, and glomerular endotheliosis in pregnant rats. Maternal serum levels of sflt-1 have been found significantly elevated in PE compared with controls and correlated with the severity of the disease. It suggested that excess circulating sflt- 1 may contribute to the pathology of PE $[1,3,21]$. In contrast, VEGF and PlGF have been reported decreased in PE patients [22] As sflt-1, alternations of levels of VEGF and PIGF are shown prior the onset of the symptoms. Unlike sflt-1, VEGF and PIGF alternations can be found at the end of the first trimester of pregnancy complicated with PE. VEGF has also been confirmed to be associated with origin of the disease $[22,23]$. In a study of mice, a half cut off of VEGF expression could result in proteinuria and glomerular endothelial injury. In human, antagonists of VEGF in renal cell carcinoma may lead to hypertension and proteinuria [1,24]. Angiogenic factors have already regarded as candidate biomarkers for PE [25]. However, a single marker can not fulfill the task, there is a need for a series of markers to achieve the goals. According to the previous study, the optimal cut-off value of the $\log [\mathrm{sFlt}-1 / \mathrm{PlGF}]$ ratio was significantly higher in PE than controls [1,26]. It has been suggested that higher levels of sflt-1 combined with lower PLGF 
may be more effective in prediction the development of PE. Further studies with larger population are required to assess its clinical value.

\subsection{PP-13}

Placental protein 13 (PP-13) is a galectin expressed by the syncytiotrophoblast, locating at the maternal-fetal interface. It is thought to be related with placental invasion and maternal remodeling [27]. According to the previous studies, it was demonstrated that PP-13 was significantly decreased in the first trimester of women who later developed $\mathrm{PE}$ and fetus growth restriction (FGR). Therefore, PP-13 was not regarded as a specific marker for PE. According to further studies, PP-13 was confirmed up-regulated at $9-12$ weeks of gestation $[28,29]$. But Renate Hillermann reported that the downregulated levels of PP-13 might be related to the deletion frame-shift mutation of LGALS13 gene, the mutant PP-13 protein cannot be recognized by existing monoclonal anti-PP13 antibodies, which might result in the lower levels of PP13 in the first trimester of pregnancy complicated with PE [30]. Combing with uterine artery Doppler, the predictive value of PP-13 seems to be improved [28]. However, whether PP-13 used alone, or combing with uterine artery Doppler appears to be a promising area for future research in this field.

\section{MATERNAL INFLAMMATORY FACTORS}

Kinds of inflammatory cytokines have been believed to be involved in inadequate trophoblast invasion and spiral artery remodeling, which may induce imbalance of a maternal inflammatory response. It has been demonstrated that the aberrant lymphocyte Th1/Th2 cytokine balance could be associated with the pathophysiology changes of PE. Th1-type cytokines, such as $\alpha$-tumoral necrosis factor (TNF- $\alpha$ ), $\gamma$-interferon (IFN- $\gamma$ ) and interleukin 2 (IL-2), can induce an exaggerated maternal systemic inflammatory response which may lead to strong maternal response that causes hypertension and proteinuria [31,32]. TNF- $\alpha$, as a Th1-type cytokines, released from a variety of cell types on appropriate stimulation, including trophoblast cells, contributes to the trophoblast growth and invasion. According to the study of Michael Cackovic, urinary concentrations of TNF- $\alpha$ were significantly decreased in PE, indicated it may contribute to the exaggerated inflammatory response observed in PE. Tai-Ho hung has reported that the ischemic placenta could release higher TNF- $\alpha$ compared to controls, implied that increased secretion of TNF- $\alpha$ may contribute to damages of maternal endothelial cells. Consistently, reported by Michael Cackovic, serum concentrations of TNF- $\alpha$ were significantly higher in PE women. Future work should allow its confirmation [33,34]. Th2 cytokines such as interleukin 6 (IL-6), interleukin 10 (IL-10) can stimulate the differentiation and proliferation of the trophoblast [35]. Th2-type activity plays a crucial role during a promoting successful pregnancy. Imbalance of Th1/Th2 cytokines may contribute to origin of PE. High levels of Th1 and low levels of Th2 cytokines could lead to a functional injury of trophoblast in PE. IL-6, a Th2type cytokines regulate acute phase reaction, interferes with endothelial cell function, modulates TNF- $\alpha$ production, also contributes to the trophoblast growth and invasion [31,32]. According to the previous work, IL-6 and IL-10 was decreased in PE patients compared with controls. On the contrary, study of J Tavakkol Afshari revealed that IL-6 levels were significantly higher in PE women [36,37]. However, changes of IL-6 are not stable in PE, whether it can be biomarker for PE remains uncertain.

\section{METABOLIC RELATED FACTORS}

Significant metabolic alternations can be demonstrated in PE. Some metabolic factors may participant in the pathology changes or serve as results of the pathology variation.

\subsection{PAPP-A}

Pregnancy-associated plasma protein A (PAPP-A, pappalysin 1, insulin-like growth factor binding protein-4 protease) is a glycosylated protein complex produced by the developing trophoblast, and has already been used in as a marker for Downs' syndrome [38]. PAPP-A was first shown to be elevated in the plasma of PE in 1980 [39]. However, according to the recent studies, plasma levels of PAPP-A was shown decreased in all three trimesters in women with $\mathrm{PE}$, indicated that it may be associated with the placenta pathology changes of PE [1]. While lower concentrations are also presented in other complications of pregnancy, such as FGR. Canini maintained that PAPP-A was more useful as a marker for FGR than PE [40]. Furthermore, a correlation between birth weight and maternal PAPP-A plasma levels have been reported. Suggested that PAPP-A might be correlated with placenta ischemia in these complication of pregnancy [41]. However, similar to PP-13, the combination of uterine artery Doppler may increase its sensitivity to the prediction of $\mathrm{PE}$.

\subsection{Transthyretin}

Transthyretin (TTR) is synthesized mainly in the liver, eye and choroid plexus, belongs to a group of proteins including thyroxine-binding globulin and albumin which bind and transport thyroid hormones in the blood, and its 
main function is the transport of thyroxin $\left(\mathrm{T}_{4}\right)$ [42]. TTR is also synthesized by placental trophoblasts, which is critical for the normal development of fetus. TTR secreted from placenta has been proved to play crucial role in transference of maternal thyroid hormone to the fetal circulation though TTR-T4 complex for fetus development [43]. According to the recent researches, TTR was found decreased in maternal serum of women with PE $[44,45]$. As the central pathology changes in PE, placenta necrosis could lead to decrease secretion of TTR, which might result in disorders of fetus development in severe PE. However, whether levels of TTR can change in women with FGR remains unclear. The question why TTR protein was decreased in PE women has not been revealed. Whether TTR can become a new potential biomarker remains unknown. There is a need for a larger scale confirmative studies to achieve the goals. Further study is worth performing.

\section{CONCLUSIONS}

To date, the precise origin of $\mathrm{PE}$ is believed to be multifactorial and has not been clarified yet. But placenta has already confirmed to be the central place in pathology changes of PE. A long standing hypothesis has been that inadequate transformation of uterine spiral arteries and subsequently worsened placental perfusion [1-3]. Alternations of a series of maternal factors could lead to the pathology changes of PE [1,4].

Despite the development of obstetrics and neonatal care have led to a decrease in maternal and fetal/neonatal mortality and morbidity of PE. The ability in prediction and early diagnosis of the disease have not significantly improved, clinicians still rely on a regular antenatal care before deliveries. Patients with PE can only be diagnosis and get treated once they have developed classical manifestations, when the treatment options have been limited. There is a great need for the development of predictive tools for PE $[1,2,46]$.

We here present a series of factors that correlated with placenta pathology. The factors released from placenta of women with PE may be associated with pathology changes of PE. Some of the factors may be confirmed to be useful biomarkers in the early prediction and monitoring of the disease in a future.

Attention has turned to identifying potential biomarkers for the early diagnosis of the disease [1]. However, the demonstration for the proteins different expressed in PE is far from working as a predictor. There is a need for high quality, large scale multicenter trials which can assess the predictive value of different markers and clarify pathogenesis of the disease $[1,46]$.

It is hoped that the new technology will help us find pathophysiology changes of PE and explore biomarkers with high predictive and prognosis values to be used into clinical practice. We hope it will not be distant from today.

\section{REFERENCES}

[1] Simon, G., Corinne, R., Rosanna, Z.-D., Sevgi, T., Wolfgang, H., Sinuhe, H. and Olav, L. (2009) Potential markers of preeclampsia: A review. Reproductive Biology and Endocrinology, 7, 70. doi:10.1186/1477-7827-7-70

[2] Franziska, P. and Mark, A.B. (2012) The management of pre-eclampsia: What we think we know. European Journal of Obstetrics \& Gynecology and Reproductive Biology, 160, 6-12. doi:10.1016/j.ejogrb.2011.09.049

[3] Hacker, G.H. (2010) Hacker and Moore's essentials of obstetrics and gynecology. 5th Edition, Sunders.

[4] Gustaaf, A., Dekker, G., Baha, M. and Sibai, B. (1998) Etiology and pathogenesis of preeclampsia: Current concepts. American Journal of Obstetrics \& Gynecology, 179, 1359-1375. doi:10.1016/S0002-9378(98)70160-7

[5] Hertig, A. and Liere, P. (2010) New markers in preeclampsia. Clinica Chimica Acta, 411, 1591-1595. doi:10.1016/j.cca.2010.07.020

[6] Julie, A.W., Johnson B.L., Cam, T.H. and Gabriela, S.D. (2008) Murine pregnancy-specific glycoprotein 23 induces the proangiogenic factors transforming-growth factor beta 1 and vascular endothelial growth factor a in cell types involved in vascular remodeling in pregnancy. Biology of Reproduction, 79, 1054-1061. doi:10.1095/biolreprod.108.070268

[7] Melissa, L.W., Daniel, H.D., Murphy, T.G., David, A.M. and Sue, A.I. (2009) Maternal and fetal variants in the TGF-beta3 gene and risk of pregnancy-induced hypertension in a predominantly latino population. American Journal of Obstetrics \& Gynecology, 201, 295.e1-295.e5.

[8] Maynard, S., Epstein, F.H. and Karumanchi, S.A. (2008) $\mathrm{PE}$ and angiogenic imbalance. Annual Review of Medicine, 59, 61-78. doi:10.1146/annurev.med.59.110106.214058

[9] Venkatesha, S., Toporsian, M., Lam, C., Hanai, J., Mammoto, T., Kim, Y.M., Bdolah, Y., Lim, K.H., Yuan, H.T., Libermann, T.A., Stillman, I.E., Roberts, D., D’Amore, P.A., Epstein, F.H., Sellke, F.W., Romero, R., Sukhatme, V.P., Letarte, M. and Karumanchi, S.A. (2006) Soluble endoglin contributes to the pathogenesis of preeclampsia. Nature Medicine, 12, 642-649. doi:10.1038/nm1429

[10] Stepan, H., Geipel, A., Schwarz, F., Kramer, T., Wessel, N. and Faber, R. (2008) Circulatory soluble endoglin and its predictive value for preeclampsia in second-trimester pregnancies with abnormal uterine perfusion. American Journal of Obstetrics \& Gynecology, 198, 175-176. doi:10.1016/j.ajog.2007.08.052

[11] Romero, R., Nien, J.K., Espinoza, J., Todem, D., Fu, W., Chung, H., Kusanovic, J.P., Gotsch, F., Erez, O., MazakiTovi, S., Gomez, R., Edwin, S., Chaiworapongsa, T., Levine, R.J. and Karumanchi, S.A. (2008) A longitudinal study of angiogenic (placental growth factor) and antiangiogenic (soluble endoglin and soluble vascular endo- 
thelial growth factor receptor-1) factors in normal pregnancy and patients destined to develop preeclampsia and deliver a small for gestational age neonate. Journal of Maternal-Fetal and Neonatal Medicine, 21, 9-23. doi:10.1080/14767050701830480

[12] Jeyabalan, A., McGonigal, S., Gilmour, C., Hubel, C.A. and Rajakumar, A. (2008) Circulating and placental endoglin concentrations in pregnancies complicated by intrauterine growth restriction and preeclampsia. Placenta, 29, 555-563. doi:10.1016/j.placenta.2008.03.006

[13] Stepan, H., Kramer, T. and Faber, R. (2007) Maternal plasma concentrations of soluble endoglin in pregnancies with intrauterine growth restriction. Journal of Clinical Endocrinology \& Metabolism, 92, 2831-2834. doi:10.1210/jc.2006-2774

[14] Luisi, S., Florio, P., Reis, F.M. and Petraglia, F. (2005) Inhibins in female and male reproductive physiology: Role in gametogenesis, conception, implantation and early pregnancy. Human Reproduction Update, 11, 123-135. doi:10.1093/humupd/dmh057

[15] Muttukrishna, S., North, R.A., Morris, J., Schellenberg, J.C. and Taylor, R.S. (2000) Serum inhibin A and activin A are elevated prior to the onset of pre-eclampsia. Human Reproduction, 15, 1640-165. doi:10.1093/humrep/15.7.1640

[16] Aparna, R., Sangeeta, S., Ian, L.S., Christopher, W.G. and Shanthi, M. (2009) Maternal Circulating levels of Activin A, Inhibin A, sFlt-1 and endoglin at parturition in normal pregnancy and pre-eclampsia. PLoS ONE, 4, e4453. doi:10.1371/journal.pone.0004453

[17] Shin-Young, K., Hyun-Mee, R., Jae-Hyug, Y., MoonYoung, K., Hyun-Kyong, A., Joong-Sik, S., Jun-Seek, C., So-Yeon, P., Jin-Mi, K., Bom-Yi, L., Do-Jin, K. (2006) Maternal serum and amniotic fluid Inhibin A levels in women who subsequently develop severe preeclampsia. Journal of Korean Medical Science, 21, 452-456. doi:10.3346/jkms.2006.21.3.452

[18] Ahmed, A., Li, X.F., Dunk, C., Whittle, M.J., Rushton, D.I. and Rollason, T. (1995) Colocalisation of vascular endothelial growth factor and its Flt-1 receptor in human placenta. Growth Factors, 12, 235-243. doi:10.3109/08977199509036883

[19] Khaliq, A., Li, X.F., Shams, M., Sisi, P., Acevedo, C.A., Whittle, M.J., Weich, H. and Ahmed, A. (1996) Localisation of placenta growth factor (PIGF) in human term placenta. Growth Factors, 13, 243-50. doi:10.3109/08977199609003225

[20] Ong, S., Lash, G. and Baker, P.N. (2000) Angiogenesis and placental growth in normal and compromised pregnancies. Best Practice \& Research Clinical Obstetrics \& Gynaecology, 14, 969-980. doi:10.1053/beog.2000.0138

[21] Yelumalai, S., Muniandy, S., Zawiah, O.S. and Qvist, R. (2010) Pregnancy-induced hypertension and preeclampsia. Levels of angiogenic factors in malaysian women. Journal of Clinical Biochemistry and Nutrition, 47, 191197. doi:10.3164/jcbn.10-27

[22] Wikstrom, A.K., Larsson, A., Eriksson, U.J., Nash, P., Norden-Lindeberg, S. and Olovsson, M. (2007) Placental growth factor and soluble FMS-like tyrosine kinase-1 in early-onset and late-onset preeclampsia. Obstetrics \& Gynecology, 109, 1368-1374.

doi:10.1097/01.AOG.0000264552.85436.a1

[23] Chaiworapongsa, T., Romero, R., Kim, Y.M., Kim, G.J., Kim, M.R., Espinoza, J., Bujold, E., Goncalves, L., Gomez, R., Edwin, S. and Mazor, M. (2005) Plasma soluble vascular endothelial growth factor receptor-1 concentration is elevated prior to the clinical diagnosis of preeclampsia. Journal of Maternal-Fetal and Neonatal Medicine, 17, 3-18. doi:10.1080/14767050400028816

[24] EunSung, L., Min-Jeong, O., JaeWon, J., Ji-Eun, L., HyunJoo, S., Kyung-Ju, L. and Hai-Joong, K. (2007) The levels of circulating vascular endothelial growth factor and soluble Flt-1 in pregnancies complicated by preeclampsia. Journal of Korean Medical Science, 22, 94-98. doi:10.3346/jkms.2007.22.1.94

[25] Gastrich, M.D., Faro, R. and Rosen, T. (2010) Markers of preeclampsia and the relationship to cardiovascular disease. Review of the twenty-first century literature. Journal of Maternal-Fetal and Neonatal Medicine, 23, 751769. doi:10.3109/14767058.2010.481316

[26] Verlohren, S., Galindo, A., Schlembach, D., Zeisler, H., Herraiz, I., Moertl, M.G., Pape, J., Dudenhausen, J.W., Denk, B. and Stepan, H. (2010) An automated method for the determination of the sFlt-1/PIGF ratio in the assessment of preeclampsia. American Journal of Obstetrics \& Gynecology, 202, 161.e1-161.e11.

[27] Nicolaides, K.H., Bindra, R. and Turan, O.M. (2006) A novel approach to first-trimester screening for early preeclampsia combining serum PP-13 and Doppler ultrasound. Ultrasound in Obstetrics \& Gynecology, 27, 13-17. doi:10.1002/uog.2686

[28] Burger, O., Pick, E. and Zwickel, J. (2004) Placental protein 13 (PP-13): Effects on cultured trophoblasts, and its detection in human body fluids in normal and pathological pregnancies. Placenta, 25, 608-622. doi:10.1016/j.placenta.2003.12.009

[29] Chafetz, I., Kuhnreich, I. and Sammar, M. (2007) Firsttrimester placental protein 13 screening for preeclampsia and intrauterine growth restriction. American Journal of Obstetrics \& Gynecology, 197, 35.e1-35.e7.

[30] Nandor, G.T., Roberto, R., Renate, H., Veronica, C., Guiying, N. and Berthold, H. (2008) Prediction of preeclampsia-A workshop report. Placenta, 29, S83-S85. doi:10.1016/j.placenta.2007.10.008

[31] Hernan, D., Kopcow, S. and Ananth, K. (2007) Angiogenic factors and Natural Killer (NK) cells in the pathogenesis of preeclampsia. Journal of Reproductive Immunology, 76, 23-29. doi:10.1016/j.jri.2007.03.018

[32] Norma, C.S. (2006) Immunology and genetic of preeclampsia. Clinical \& Developmental Immunology, 13, 197-201. doi:10.1080/17402520600876903

[33] Tai-Ho, H., Charnock-Jones, D.S., Jeremy, N.S. and Graham, J.B. (2004) Secretion of tumor necrosis factor from human placental tissues induced by hypoxia-reoxygenation causes endothelial cell activation in vitro: A potential mediator of the inflammatory response in preeclam psia. American Journal of Pathology, 164, 1049-1061. doi:10.1016/S0002-9440(10)63192-6 
[34] Michael, C., Catalin, S.B., Guomao, Z., Edmund, F.F., Errol, R., Norwitz, E.K, Charles, J.L. and Irina, A.B. (2008) Fractional excretion of tumor necrosis factor- $\alpha$ in women with severe preeclampsia. Obstetrics \& Gynecology, 112, 93-100. doi:10.1097/AOG.0b013e31817c4304

[35] Charles, J.L., Chih-Feng, Y., Murat, B., Umit, A., Kayisli, M.M., Irina, B., Catalin, B.S., Joseph, H., Graciela, K. and Frederick, S. (2008) Preeclampsia-related inflammatory cytokines regulate interleukin-6 expression in human decidual cells. The American Journal of Pathology, 172, 1571-1579. doi:10.2353/ajpath.2008.070629

[36] Jacek, R., Wilczynski, H.T., Ewa, G., Malgorzata, B., Przemyslaw, L., Marian, S., Krzysztof, Z. and Jan, W. (2002) Cytokine secretion by decidual lymphocytes in transient hypertension of pregnancy and pre-eclampsia. Mediators of Inflammation, 11, 105-111. doi:10.1080/09629350220131962

[37] Tavakkol, A.J., Ghomian, N., Shameli, A., Shakeri, M.T., Fahmidehkar, M.A., Mahaje, E., Khoshnavaz, R. and Emadzadeh, M. (2005) Determination of Interleukin-6 and tumor necrosis factor-alpha concentrations in iranian-khorasanian patients with preeclampsia. BMC Pregnancy and Childbirth, 5, 14. doi:10.1186/1471-2393-5-14

[38] Bersinger, N.A., Smarason, A.K. and Muttukrishna, S. (2003) Women with preeclampsia have increased serum levels of pregnancy-associated plasma protein a (PAPPA), inhibin A, activin A, and soluble E-selectin. Hypertens Pregnancy, 22, 45-55. doi:10.1081/PRG-120016794

[39] Hughes, G., Bischof, P., Wilson, G. and Klopper, A. (1980) Assay of a placental protein to determine fetal risk. $B M J$, 280, 671-673. doi:10.1136/bmj.280.6215.671

[40] Canini, S., Prefumo, F. and Pastorino, D. (2008) Associa- tion between birth weight and first-trimester free betahuman chorionic gonadotropin and pregnancy-associated plasma protein A. Fertility and Sterility, 89, 174-178. doi:10.1016/j.fertnstert.2007.02.024

[41] Toop, K. and Klopper, A. (1981) Concentration of pregnancy-associated plasma protein A (PAPP-A) in patients with pre-eclamptic toxaemia. Placenta Supplement, 3, 167-173.

[42] Fleming, C.E., Nunes, A.F. and Sousa, M.M. (2009) Transthyretin: More than meets the eye. Progress in Neurobiology, 89, 266-276. doi:10.1016/j.pneurobio.2009.07.007

[43] Landers, K.A., McKinnon, B.D., Li, H., Subramaniam, V.N., Mortimer, R.H. and Richard, K. (2009) Carriermediated thyroid hormone transport into placenta by placental transthyretin. Journal of Clinical Endocrinology \& Metabolism, 94, 2610-2616. doi:10.1210/jc.2009-0048

[44] Ulrich, P., Franka, S., Claudia, R., Toralf, R., Werner, R. and Michael, O.G. (2010) Multifactorial analysis of affinity-mass spectrometry data from serum protein samples: A strategy to distinguish patients with preeclampsia from matching control individuals. Journal of the American Society for Mass Spectrometry, 21, 1699-1711. doi:10.1016/j.jasms.2009.12.013

[45] Liu, C., Zhang, N., Yu, H., Chen, Y., Liang, Y., Deng, H. and Zhang, Z. (2011) Proteomic analysis of human serum for Finding pathogenic factors and potential biomarkers in preeclampsia. Placenta, 32, 168-174. doi:10.1016/j.placenta.2010.11.007

[46] David, M.C., Christian, D. and Anna, F.D. (2008) Novel biomarkers for predicting preeclampsia. Trends in Cardiovascular Medicine, 18, 186-194. doi:10.1016/j.tcm.2008.07.002 